\title{
ESTIMATING THE STATE OF A NOISY CONTINUOUS TIME MARKOV CHAIN WHEN DYNAMIC SAMPLING IS FEASIBLE
}

\author{
By DAVID ASSAF
}

\author{
The Hebrew University
}

\begin{abstract}
A continuous time Markov chain is observed with Gaussian white noise added to it. To the well-known problem of continuously estimating the current state of the chain, we introduce the additional option of continuously varying the sampling rates, as long as some restriction (or cost) on the average sampling rate is satisfied. The optimal solution to this "dynamic sampling" problem is presented and analyzed in closed form for the two-state symmetric case. It is shown that the resulting dynamic sampling procedure has a much lower asymptotic average error rate compared to the one obtained when sampling at a constant rate. Alternatively, the dynamic sampling procedure can provide the same error rate using a much lower average sampling rate. The relative efficiency of the dynamic sampling procedure may in fact tend to infinity in some extreme cases.
\end{abstract}

1. The basic problem. Let $\{Y(t) ; t \geq 0\}$ be a continuous time Markov chain with a finite number of states and some known initial condition. Assume that the actual state of the chain is observed with an added Gaussian white noise having a known variance coefficient $\sigma^{2}(\sigma>0)$. Thus, using the conventional integrated form, the observed process $\{Z(t) ; t \geq 0\}$ is given by

$$
Z(t)=\int_{0}^{t} Y(s) d s+\sigma W(t),
$$

where $W(t)$ is standard Brownian motion, which is assumed to be independent of the $Y$-process. The basic problem considered here is a continuous estimation of the true state $Y(t)$, based on the observed process $Z(t)$, with the objective of minimizing the long run average error rate.

Problems of this type occur naturally in many applications. To fix ideas consider the following examples.

1. Quality control problems: specifically, oil flowing in a pipeline where $Y(t)$ is the actual quality at time $t$ while $Z(t)$ reflects our information regarding the quality based on continuously sampling, say, 2 percent of the oil flow (a similar example occurs when testing for air quality using a test tube).

2. Communication systems (telegraph processes) with $Y(t)$ being the signal, possibly the index of the station which is currently transmitting, and $W(t)$ the noise caused by inaccuracies of the satellite radars.

3. Geological or oceanographic studies: a typical situation is one in which several types of layers are possible, and the objective is to "guess" the correct

Received October 1996; revised January 1997.

AMS 1991 subject classifications. 62M20, 93E20, 60J27, 60J60.

Key words and phrases. Filtering, dynamic sampling, Gaussian white noise, diffusion process, optimal control, average error rate. 
type at every point, based on measurements taken continuously throughout the layers. In this particular example the variable $t$ evidently relates to distance or depth rather than time, while the noise is a result of measurement sampling error.

The problem described at the beginning of this section is often referred to as a filtering problem and has been studied, together with many of its extensions, throughout the years. For basic references see Liptser and Shiryayev (1977) and the references cited there. Among the more recent references, we mention the comparison between filtering and smoothing studied by Yao (1985) and a simplified approximation method for the problem by Khasminskii and Lasareva (1992).

In this article we deal with the special case in which the $Y$-process has only two possible states, say 0 and 1 , with known and equal transition rates denoted by $\lambda(\lambda>0)$ between them. Some of the results obtained remain valid in more general setups, but the computations involved in most cases seem to be intractable and are not the main issue of this article. For a further discussion on possible extensions, see comment 7 , Section 5 .

A rigorous presentation and solution of the problem is given in Liptser and Shiryayev (1977) and several of the derivations there are used in the present article. A brief summary of the problem and its solution is the following: let $\mathscr{F}_{t}$ be the $\sigma$-field generated by $\{Z(s): 0 \leq s \leq t\}$. An estimator $\hat{Y}$ of $\{Y(t) ; t \geq 0\}$ is any $\mathscr{T}_{t}$-adapted process $\{\hat{Y}(t) ; t \geq 0\}$ (i.e., $\hat{Y}(t)$ is $\mathscr{F}_{t}$-measurable). For any estimator $\hat{Y}$, define the long-run average error rate as

$$
\alpha(\hat{Y})=\lim _{t \rightarrow \infty} \frac{1}{t} \int_{0}^{t} I(\hat{Y}(s) \neq Y(s)) d s,
$$

where we agree to replace the lim by lim sup whenever needed throughout the paper. The objective is to minimize the error rate $\alpha(\hat{Y})$ over all possible estimators $\hat{Y}$.

The solution is fairly straightforward: let $X(t)=p\left(Y(t)=1 \mid \mathscr{F}_{t}\right)$ be the posterior probability that the actual state is 1 , given all "current" accumulated information. The process $\{X(t) ; t \geq 0\}$ is sufficient for the problem and we henceforth consider the problem in terms of this $X$-process only. Estimate $Y(t)$ by

$$
\hat{Y}^{*}(t)= \begin{cases}1, & X(t) \geq \frac{1}{2}, \\ 0, & X(t)<\frac{1}{2} .\end{cases}
$$

The estimator $\hat{Y}^{*}$ in (1.3) leads to the expression for the long-run average error rate as

$$
\alpha\left(\hat{Y}^{*}\right)=\lim _{t \rightarrow \infty}\left[\frac{1}{t} \int_{0}^{t} \min (X(s), 1-X(s)) d s\right] .
$$

It turns out that the limit in (1.4) exists and may be computed in a fairly explicit way using the stationary distribution of $X(t)$ (see Section 4). The value 
of $\alpha\left(\hat{Y}^{*}\right)$ in (1.4) is the lowest long-run average estimation error attainable, that is, $\alpha\left(\hat{Y}^{*}\right)=\inf \{\alpha(\hat{Y})\}$ with the infimum taken over all estimators $\hat{Y}$.

2. Dynamic sampling formulation. The dynamic sampling version of the problem utilizes the following idea: suppose that we are allowed to vary the sample size as we go along, rather than sampling at the same rate all the time. In example (1) this will perhaps mean channeling only 1 percent of the inflowing oil for quality control examination in some instances while channeling 5 percent (or 20 percent or 36 percent) in other instances, rather than keeping the rate at the constant level of 2 percent all the time. In example (2) we may want to vary the number of radars "pointed" at each of the stations (or vary the computational effort allocated to each of them), and in example (3) we may sample a different amount of sediment at each depth.

The mathematical model for the dynamic sampling formulation is as follows: think of $\sigma^{2}$ in the basic model as the variance coefficient of the Gaussian white noise when sampling at some standard fixed rate of unity [e.g., 2 percent in example (1)]. When sampling at rate $u(u>0)$ for $\Delta t$ time units, the accumulated amount sampled is $u \Delta t$ units (compared to $\Delta t$ in the fixed rate basic model) resulting in an instantaneous variance coefficient of $\sigma^{2} / u$ (see comment 8 , Section 5). A sampling procedure $U$ is defined as a nonnegative process $\{U(t) ; t \geq 0\}$ which is adapted to $\left\{\tilde{\mathscr{F}_{t}} ; t \geq 0\right\}$ where $\tilde{\mathscr{F}}_{t}$ is the $\sigma$-field generated by $\{X(s) ; 0 \leq s \leq t\}$ and $\{U(s) ; 0 \leq s<t\}$. Thus a decision to sample at rate $u$ from time $t$ to time $t+\Delta t$ may depend on the history of the $X$-process up to time $t$ as well as on the sampling rates selected during the time interval $[0, t)$. Since $U$ affects the stochastic behavior of the $X$-process, the problem is evidently an optimal control one and is indeed analyzed as such.

Two more preliminary aspects are needed before a rigorous formulation of the dynamic sampling problem can be made. The first is noting that it is necessary to put some constraint or cost on the sampling rates (otherwise one would always choose the highest rate possible, thereby trivializing the problem). For most of the paper we impose a cost of $c$ per unit of sampling. The constrained version is equivalent (see Section 4). The second preliminary result needed is the behavior of the $X$-process. A slight extension of the derivation in Liptser and Shiryayev (1977) shows that $\{X(t) ; t \geq 0\}$ is a diffusion process taking values in $[0,1]$ with instantaneous drift and variance coefficients given by

$$
\begin{gathered}
\mu(x)=\lambda(1-2 x), \\
\sigma^{2}(x)=u \frac{x^{2}(1-x)^{2}}{\sigma^{2}},
\end{gathered}
$$

where $x$ is the current state of the process and $u$ is the instantaneous sampling rate applied. It is worthwhile to point out that (2.2) holds for $u=0$ as well. In this case no sampling is done, hence no noise is present in the system and the $X$-process moves deterministically according to its drift. 
The dynamic sampling problem is to determine both a sampling procedure and an estimator to minimize the long-run average cost per unit time, where the cost is composed of cost per error and per sampling costs. Thus for any sampling procedure $U$ and estimator $\hat{Y}$ define

$$
V(U, \hat{Y})=\lim _{t \rightarrow \infty}\left\{\frac{1}{t}\left[\int_{0}^{t} I(\hat{Y}(s) \neq Y(s)) d s+c \int_{0}^{t} U(s) d s\right]\right\}
$$

to be the long-run average cost per unit time when using $U$ and $\hat{Y}$, and define

$$
v^{*}=\inf V(U, \hat{Y})
$$

with the infimum taken over all choices of $U$ and $\hat{Y}$. The objective is to evaluate $v^{*}$ and, if possible, to find a pair $(U, \hat{Y})$ which is optimal in the sense of attaining the infimal value.

For $\hat{Y}$ equal to $\hat{Y}^{*}$ of (1.3), expression (2.3) may equivalently be written as

$$
V\left(U, \hat{Y}^{*}\right)=\lim _{t \rightarrow \infty}\left\{\frac{1}{t}\left[\int_{0}^{t} \min (X(s), 1-X(s)) d s+c \int_{0}^{t} U(s) d s\right]\right\} .
$$

The estimator $\hat{Y}^{*}$ is indeed the optimal one for the dynamic sampling version as well, and the main focus of the paper is hence on the sampling rate and the performance of the dynamic sampling procedure. Use of $\hat{Y}^{*}$ is in fact assumed whenever $\hat{Y}$ is not mentioned explicitly. The proof of optimality in Section 3 does indicate the proof of optimality of $\hat{Y}^{*}$ as a by-product (see also comment 3 , Section 5).

Since $\hat{Y}^{*}$ is optimal and depends on the history only through the current value of $X$, we have simplified the filtration $\left\{\tilde{\mathscr{F}}_{t}\right\}$ slightly. A totally rigorous formulation (which would make no difference in the analysis) would require that the pair $(U(t), \hat{Y}(t))$ be adapted to the $\sigma$-field generated by $\{X(s) ; 0 \leq$ $s \leq t\},\{U(s) ; 0 \leq s<t\}$ and $\{Y(s) ; 0 \leq s<t\}$.

3. Main results. Our main result in this section is the explicit evaluation of $v^{*}$, the infimal average cost per unit time for the dynamic sampling version of the problem as defined in (2.4). The first theorem establishes a lower bound on $v^{*}$.

THEOREM 1. Denote

$$
l^{*}=\inf _{0 \leq x \leq \frac{1}{2}}\left\{x+2 \lambda \sigma^{2} c(1-2 x)\left[2 \ln \left(\frac{1-x}{x}\right)+\frac{1-2 x}{x(1-x)}\right]\right\} .
$$

Then

$$
v^{*} \geq l^{*}
$$

The following technical lemma is needed. 
LEMMA 1. (i) The function

$$
g(x)=\frac{x^{2}(1-x)^{2}}{(1-2 x)^{2}}
$$

is strictly increasing on $\left[0, \frac{1}{2}\right)$ with $g(0)=0$ and $g(x) \rightarrow \infty$ as $x \rightarrow \frac{1}{2}$.

(ii) The function

$$
h(x)=x+b(1-2 x)\left[2 \ln \left(\frac{1-x}{x}\right)+\frac{1-2 x}{x(1-x)}\right]
$$

for any fixed $b>0$, is strictly convex on $\left(0, \frac{1}{2}\right]$, satisfies $h(x) \rightarrow \infty$ as $x \rightarrow$ $0, h\left(\frac{1}{2}\right)=\frac{1}{2}$ and its unique minimum over $\left[0, \frac{1}{2}\right]$ is attained at some inner point $0<\alpha<\frac{1}{2}$.

PROOF. The proof follows from direct examination of the appropriate derivatives. Convexity of $h(x)$ is perhaps easier than may be anticipated since the second derivative simplifies to

$$
h^{\prime \prime}(x)=2 \cdot b \frac{2 x^{2}-2 x+1}{x^{3}(1-x)^{3}},
$$

while the last statement regarding $h(x)$ follows from its strict convexity together with $h(x) \rightarrow \infty$ as $x \rightarrow 0$ and $h^{\prime}\left(\frac{1}{2}\right)>0$.

Let $l(x)$ be equal to $h(x)$ of (3.4) for $b=2 \lambda \sigma^{2} c$ and let $0<\alpha^{*}<\frac{1}{2}$ be the unique minimizer of $l(x)$ in $\left[0, \frac{1}{2}\right]$. Thus $l^{*}=l\left(\alpha^{*}\right)$ is the quantity defined in (3.1).

Proof OF THEOREM 1. Applying standard results in dynamic programming and control (see comments 3 and 4 in Section 5), it suffices to show the existence of a function $G(x)$ which is twice continuously differentiable over $[0,1]$ and which satisfies the inequality

$$
l^{*} \leq \min \{x, 1-x\}+c \cdot u+L(u) G(x),
$$

where

$$
L(u) G(x)=\lambda(1-2 x) G^{\prime}(x)+\frac{1}{2} \cdot u \cdot \frac{x^{2}(1-x)^{2}}{\sigma^{2}} G^{\prime \prime}(x)
$$

is the differential operator corresponding to sampling at rate $u$. The coefficients of $G^{\prime}(x)$ and $\frac{1}{2} G^{\prime \prime}(x)$ are the corresponding drift and variance given in (2.1) and (2.2). Inequality (3.5) needs to hold for all $0 \leq x \leq 1$ and $u \geq 0$.

We next exhibit a function $G$ satisfying all the requirements needed. For the heuristic interpretation of $G$ see comment 5 , Section 5 . To define the function $G$, begin with the following two functions:

$$
G_{0}(x)=\frac{1}{2 \lambda}\left[\left(x-\alpha^{*}\right)+\left(\frac{1}{2}-l^{*}\right) \ln \left(\frac{1-2 x}{1-2 \alpha^{*}}\right)\right],
$$


for which

$$
\begin{aligned}
& G_{0}^{\prime}(x)=\frac{l^{*}-x}{\lambda(1-2 x)}, \\
& G_{0}^{\prime \prime}(x)=\frac{2 l^{*}-1}{\lambda(1-2 x)^{2}}
\end{aligned}
$$

and

$$
G_{1}(x)=2 c \sigma^{2}\left[(1-2 x) \ln \left(\frac{x}{1-x}\right)-\left(1-2 \alpha^{*}\right) \ln \left(\frac{\alpha^{*}}{1-\alpha^{*}}\right)\right]
$$

so that

$$
\begin{gathered}
G_{1}^{\prime}(x)=2 c \sigma^{2}\left[2 \ln \left(\frac{1-x}{x}\right)+\frac{1-2 x}{x(1-x)}\right] \\
G_{1}^{\prime \prime}(x)=\frac{-2 c \sigma^{2}}{x^{2}(1-x)^{2}} .
\end{gathered}
$$

Now define $G$ by

$$
G(x)= \begin{cases}G_{0}(x), & 0 \leq x \leq \alpha^{*}, \\ G_{1}(x), & \alpha^{*}<x \leq \frac{1}{2}, \\ G_{1}(1-x), & \frac{1}{2}<x \leq 1-\alpha^{*} \\ G_{0}(1-x), & 1-\alpha^{*}<x \leq 1 .\end{cases}
$$

To check that the function $G(x)$ has a continuous second order derivative in $\left[0, \frac{1}{2}\right)$, it clearly suffices to prove that

$$
\begin{aligned}
& G_{0}\left(\alpha^{*}\right)=G_{1}\left(\alpha^{*}\right), \\
& G_{0}^{\prime}\left(\alpha^{*}\right)=G_{1}^{\prime}\left(\alpha^{*}\right), \\
& G_{0}^{\prime \prime}\left(\alpha^{*}\right)=G_{1}^{\prime \prime}\left(\alpha^{*}\right) .
\end{aligned}
$$

Parts (a) and (b) follow directly from the structures of $G_{0}$ and $G_{1}$ and from the definition of $\alpha^{*}$. To prove (c), note that the function $l(x)$ (i.e., $h(x)$ of Lemma 1 , with $\left.b=2 \lambda \sigma^{2} c\right)$ may be written as $l(x)=x+\lambda(1-2 x) G_{1}^{\prime}(x)$. Since $l(x)$ is convex and has $\alpha^{*}$ as its unique minimizer in $\left(0, \frac{1}{2}\right)$, it follows that $l^{\prime}\left(\alpha^{*}\right)=0$. Now

$$
l^{\prime}(x)=1+\lambda(1-2 x) G_{1}^{\prime \prime}(x)-2 \lambda G_{1}^{\prime}(x) .
$$

Substitute $l^{\prime}\left(\alpha^{*}\right)=0, G_{1}^{\prime}\left(\alpha^{*}\right)=G_{0}^{\prime}\left(\alpha^{*}\right)$ and solve (3.14) for $G_{1}^{\prime \prime}\left(\alpha^{*}\right)$ to obtain

$$
G_{1}^{\prime \prime}\left(\alpha^{*}\right)=\frac{2 \lambda G_{0}^{\prime}\left(\alpha^{*}\right)-1}{\lambda\left(1-2 \alpha^{*}\right)}=\frac{2 \lambda\left(l^{*}-\alpha^{*}\right)-\lambda\left(1-2 \alpha^{*}\right)}{\lambda^{2}\left(1-2 \alpha^{*}\right)^{2}}
$$


so that

$$
G_{1}^{\prime \prime}\left(\alpha^{*}\right)=\frac{2 l^{*}-1}{\lambda\left(1-2 \alpha^{*}\right)^{2}}=G_{0}^{\prime \prime}\left(\alpha^{*}\right)
$$

For $\frac{1}{2} \leq x \leq 1$ we have, by the definition of $G, G(x)=G(1-x)$. Hence $G^{\prime}(x)=-G^{\prime}(1-x)$ and $G^{\prime \prime}(x)=G^{\prime \prime}(1-x)$ from which equalities analogous to (a), (b) and (c) at $1-\alpha^{*}$ follow directly. It remains to verify the behavior at $x=\frac{1}{2}$, which is easy after noting that the first derivative of $G$ at $x=\frac{1}{2}$ indeed equals 0 [otherwise the relation $G^{\prime}(x)=-G^{\prime}(1-x)$ violates the corresponding continuity at $x=\frac{1}{2}$ ].

To complete the proof of Theorem 1 we next show that $l^{*}$ and $G(x)$ satisfy inequality (3.5). Begin by proving (3.5) for $0 \leq x \leq \alpha^{*}$. In this region (3.8) implies that $G(x)=G_{0}(x)$ satisfies the equation

$$
x+\lambda(1-2 x) G^{\prime}(x)=l^{*}
$$

and it hence suffices to prove that

$$
c+\frac{1}{2} \frac{x^{2}(1-x)^{2}}{\sigma^{2}} G_{0}^{\prime \prime}(x) \geq 0
$$

or equivalently,

$$
\frac{x^{2}(1-x)^{2}}{(1-2 x)^{2}} \leq \frac{2 \lambda \sigma^{2} c}{1-2 l^{*}} .
$$

For $x=\alpha^{*}$, (3.17) holds as equality [by part (c)]. For $0 \leq x<\alpha^{*}$, (3.17) hence holds as inequality (Lemma 1 ).

For $\alpha^{*}<x \leq \frac{1}{2}, G(x)=G_{1}(x)$ and hence by (3.12) satisfies the equation

$$
c+\frac{1}{2} \frac{x^{2}(1-x)^{2}}{\sigma^{2}} G^{\prime \prime}(x)=0 .
$$

To show (3.5) it hence suffices to prove that

$$
x+\lambda(1-2 x) G_{1}^{\prime}(x) \geq l^{*} .
$$

The left-hand side of (3.19) is again $l(x)$. By the definitions of $\alpha^{*}$ and $l^{*}$, and properties of $h(x)$ (Lemma 1), (3.19) holds as equality at $x=\alpha^{*}$ and as strict inequality for any $x \neq \alpha^{*}$, thus concluding the proof for $0 \leq x \leq \frac{1}{2}$. The proof for $\frac{1}{2}<x \leq 1$ now follows by substituting $y=1-x$ and using the results obtained for $0 \leq y<\frac{1}{2}$.

Theorem 1 proves that no policy can attain a value which is strictly better than $l^{*}$. Our main result is that $l^{*}$ is in fact the value for the problem. To prove this result it would suffice to point out a policy which attains the value $l^{*}$. As far as the present author knows, however, such a policy does not exist in the strict mathematical (or "physical") sense, and the result is hence proved by introducing a family of policies which approximates $l^{*}$ in the sense that for any $\delta>0$ there exists a policy with value less than $l^{*}+\delta$. Once this family is 
introduced and analyzed to verify the approximations, we have the following main result.

THEOREM 2. The infimal average cost per unit time for the dynamic sampling problem is given by

$$
v^{*}=l^{*}
$$

with $l^{*}$ defined by (3.1).

PRoOF. Let $\alpha^{*}$ be the value minimizing $l(\alpha)$ (i.e., $l^{*}=l\left(\alpha^{*}\right)$ ), where $0<$ $\alpha^{*}<\frac{1}{2}$ is as defined in Theorem 1 and Lemma 1 .

For $0<\varepsilon<\alpha^{*}$ and $M>0$, define the sampling policy $U(M, \varepsilon)$ as follows. First, for any time at which the value of the $X$-process is $x$, the sampling rate $u$ is either 0 or $M$ according to the following:

$$
u=u(M, \varepsilon, x)= \begin{cases}M, & \alpha^{*} \leq x \leq 1-\alpha^{*}, \\ 0, & x \leq \alpha^{*}-\varepsilon \text { or } x \geq 1-\alpha^{*}+\varepsilon .\end{cases}
$$

It remains to define the sampling rate whenever the $X$-process is in the interval $\left(\alpha^{*}-\varepsilon, \alpha^{*}\right)$ [or its symmetric counterpart $\left(1-\alpha^{*}, 1-\alpha^{*}+\varepsilon\right)$ ]. This sampling rate will depend on whether the "most recent visit" of the $X$-process was at $\alpha^{*}-\varepsilon$, in which case it is 0 , or at $\alpha^{*}$, in which case it is $M$. The rate is also defined as 0 if the $X$-process has visited neither (due to initial conditions). A rigorous definition of this procedure, which also includes the symmetric $\left(1-\alpha^{*}, 1-\alpha^{*}+\varepsilon\right)$ case is given next: for any fixed $t_{0} \geq 0$ such that $\alpha^{*}-\varepsilon<X\left(t_{0}\right)<\alpha^{*}$ or $1-\alpha^{*}<X\left(t_{0}\right)<1-\alpha^{*}+\varepsilon$, define the time $0 \leq \tau_{0} \leq t_{0}$ by

$$
\tau_{0}=\left\{\begin{array}{l}
\sup \left\{s ; 0 \leq s \leq t_{0}, X(s) \in\left\{\alpha^{*}-\varepsilon, \alpha^{*}, 1-\alpha^{*}, 1-\alpha^{*}+\varepsilon\right\}\right\} \\
0, \quad \text { if no such } 0 \leq s \leq t_{0} \text { exists }
\end{array}\right.
$$

and define the sampling rate at time $t_{0}$ to be

$$
u=u\left(M, \varepsilon, t_{0}, \tau_{0}, X\left(\tau_{0}\right)\right)= \begin{cases}M, & X\left(\tau_{0}\right) \in\left\{\alpha^{*}, 1-\alpha^{*}\right\} \\ 0, & \text { otherwise }\end{cases}
$$

It should be noted that the sampling rate at time $t_{0}$ does not depend only on the current state $X\left(t_{0}\right)$, but rather depends on the history of the process $\left\{X(s) ; 0 \leq s \leq t_{0}\right\}$.

Applying the $U(M, \varepsilon)$ policy, the behavior of the $X$-process is as follows. It first reaches the value $\alpha^{*}$ (or $1-\alpha^{*}$ ) for the first time. This is a transient component which has no effect on any long-time average quantities. Once $\alpha^{*}$ or $1-\alpha^{*}$ are reached, consider cycles composed of two parts. In the first part, sampling at rate $M$ is done. This sampling is continued until the process reaches either $\alpha^{*}-\varepsilon$ or $1-\alpha^{*}+\varepsilon$, thereby completing the first part of a cycle. During the second part of a cycle, sampling is stopped, the variance coefficient of the $X$-process during this time is zero [see (2.2)] and the process moves deterministically according to its drift back to $\alpha^{*}$ (from $\alpha^{*}-\varepsilon$ ) or to 
$1-\alpha^{*}$ (from $1-\alpha^{*}+\varepsilon$ ) thereby completing one full cycle and ready to begin the next one.

Due to the cyclic nature, the long-run average cost for the $U(M, \varepsilon)$ policy may be computed using renewal-reward arguments [see Ross (1983), Chapter 3.6]. More specifically, let $C T, C S$ and $C E$ be the expected accumulated time, the expected accumulated sampling and the expected accumulated error during one cycle respectively. Then the long-run average expected cost for the $U(M, \varepsilon)$ policy denoted by $V(M, \varepsilon)$ is given by

$$
V(M, \varepsilon)=\frac{C E}{C T}+c \frac{C S}{C T} .
$$

The evaluation of the quantities $C T, C S$ and $C E$ requires some basic results in diffusion processes. A convenient reference is Karlin and Taylor (1981), Chapter 15. We begin with the evaluation of the quantities for the first part of a cycle. During this part, the $X$-process is a time homogeneous diffusion process with

$$
\frac{2 \mu(x)}{\sigma^{2}(x)}=\frac{1}{M} \frac{2 \lambda \sigma^{2}(1-2 x)}{x^{2}(1-x)^{2}} .
$$

Since $x$ is bounded away from 0 and 1, it follows that (for large $M$ ) the scale density is given by

$$
s(x)=1+O\left(\frac{1}{M}\right)
$$

Hence the scale function satisfies

$$
S(x)=x+O\left(\frac{1}{M}\right) .
$$

Due to symmetry, assume without loss of generality that the cycle begins at $\alpha^{*}$. Recall that the first part ends upon reaching $\alpha^{*}-\varepsilon$ or $1-\alpha^{*}+\varepsilon$. The Green function is hence given by

$$
H(x)=\left\{\begin{array}{l}
2 \frac{\left[S\left(\alpha^{*}\right)-S\left(\alpha^{*}-\varepsilon\right)\right]\left[S\left(1-\alpha^{*}+\varepsilon\right)-S(x)\right]}{\left[S\left(1-\alpha^{*}+\varepsilon\right)-S\left(\alpha^{*}-\varepsilon\right)\right] \sigma^{2}(x) s(x)}, \\
\alpha^{*} \leq x \leq 1-\alpha^{*}+\varepsilon, \\
2 \frac{\left[S\left(1-\alpha^{*}+\varepsilon\right)-S\left(\alpha^{*}\right)\right]\left[S(x)-S\left(\alpha^{*}-\varepsilon\right)\right]}{\left[S\left(1-\alpha^{*}+\varepsilon\right)-S\left(\alpha^{*}-\varepsilon\right)\right] \sigma^{2}(x) s(x)}, \\
\alpha^{*}-\varepsilon \leq x \leq \alpha^{*},
\end{array}\right.
$$

which simplifies to

$$
H(x)=\left\{\begin{array}{l}
\frac{2 \varepsilon\left(1-\alpha^{*}+\varepsilon-x\right) \sigma^{2}}{M\left(1-2 \alpha^{*}\right) x^{2}(1-x)^{2}}+\frac{o(\varepsilon)}{M}+\frac{O(1 / M)}{M}, \\
\frac{2\left(x-\alpha^{*}+\varepsilon\right) \sigma^{2}}{M \cdot x^{2}(1-x)^{2}}+\frac{O(\varepsilon)}{M}+\frac{O(1 / M)}{M}, \quad \alpha^{*}-\varepsilon \leq x \leq \alpha^{*} .
\end{array}\right.
$$


The three quantities for the first part of a cycle are indexed by " $a$ " and are given by

$$
\begin{gathered}
C T(a)=\int_{\alpha^{*}-\varepsilon}^{1-\alpha^{*}+\varepsilon} H(x) d x=O(\varepsilon) O\left(\frac{1}{M}\right)+o\left(\frac{1}{M}\right) \\
C S(a)=\int_{\alpha^{*}-\varepsilon}^{1-\alpha^{*}+\varepsilon} M \cdot H(x) d x \\
=\frac{2 \varepsilon \sigma^{2}}{1-2 \alpha^{*}} \int_{\alpha^{*}}^{1-\alpha^{*}} \frac{1-\alpha^{*}-x}{x^{2}(1-x)^{2}} d x+o(\varepsilon)+O\left(\frac{1}{M}\right), \\
C E(a)=\int_{\alpha^{*}-\varepsilon}^{1-\alpha^{*}+\varepsilon} \min \{x, 1-x\} \cdot H(x) d x=O(\varepsilon) O\left(\frac{1}{M}\right)+o\left(\frac{1}{M}\right) .
\end{gathered}
$$

The computations for the second part of a cycle are of different nature since during this part the process is a deterministic one. Using symmetry assume without loss of generality that the process begins at $\alpha^{*}-\varepsilon$ and ends at $\alpha^{*}$. Since the drift is $\lambda(1-2 X(t))$ and no sampling is performed, it is straightforward to evaluate the quantities, indexed by " $b$ ", for this part of a cycle. Begin with $C T(b)$. The deterministic motion may be represented as a first order linear differential equation $y^{\prime}=\lambda(1-2 y)$, the solution of which is $y(t)=A e^{-2 \lambda t}+\frac{1}{2}$, with $A$ depending on the initial condition. To solve for $C T(b)$ set $y(0)=\alpha^{*}-$ $\varepsilon, y(C T(b))=\alpha^{*}$ to obtain

$$
C T(b)=\frac{1}{2 \lambda} \ln \left(\frac{(1 / 2)-\alpha^{*}+\varepsilon}{(1 / 2)-\alpha^{*}}\right)=\frac{\varepsilon}{\lambda\left(1-2 \alpha^{*}\right)}+o(\varepsilon) .
$$

Since no sampling is performed we clearly have

$$
C S(b)=0
$$

Finally, since the $X$-process is between $\alpha^{*}-\varepsilon$ and $\alpha^{*}$ during the second part of a cycle we have

$$
\left(\alpha^{*}-\varepsilon\right) C T(b) \leq C E(b) \leq \alpha^{*} C T(b) .
$$

So that by (3.33),

$$
C E(b)=\frac{\varepsilon \alpha^{*}}{\lambda\left(1-2 \alpha^{*}\right)}+o(\varepsilon)
$$

Now $C T=C T(a)+C T(b), C S=C S(a)+C S(b)$ and $C E=C E(a)+C E(b)$. Substituting these values from (3.30), (3.31), (3.32), (3.33), (3.34) and (3.36) in the expression for the long-run average expected cost for the $U(M, \varepsilon)$ policy 
(3.24) yields

$$
\begin{aligned}
V(M, \varepsilon)= & \alpha^{*}+c 2 \sigma^{2} \lambda \int_{\alpha^{*}}^{1-\alpha^{*}} \frac{1-\alpha^{*}-x}{x^{2}(1-x)^{2}} d x \\
& +O\left(\frac{1}{M}\right)+O(\varepsilon)+O\left(\frac{1}{M}\right) O\left(\frac{1}{\varepsilon}\right) \\
= & l^{*}+O\left(\frac{1}{M}\right)+O(\varepsilon)+O\left(\frac{1}{M}\right) O\left(\frac{1}{\varepsilon}\right),
\end{aligned}
$$

where the last equality is obtained by explicit computation of the integral in (3.37). For given $\delta>0$, one may thus choose $\varepsilon>0$ small enough and $M$ large enough to yield

$$
V(M, \varepsilon) \leq l^{*}+\delta,
$$

thereby completing the proof.

4. Numerical aspects and relative efficiency. In this section we focus on the performance of the dynamic sampling procedure via the limiting value $l^{*}$ and the $U(M, \varepsilon)$ policy in the limiting $M \rightarrow \infty, \varepsilon \rightarrow 0$ sense.

(1) Recall first that $l^{*}$ is the minimum of $l(x)$ over $\left[0, \frac{1}{2}\right]$ attained at a unique $0<\alpha^{*}<\frac{1}{2}$ where

$$
l(x)=x+2 \lambda \sigma^{2} c(1-2 x)\left[2 \ln \left(\frac{1-x}{x}\right)+\frac{1-2 x}{x(1-x)}\right] .
$$

The values of $\alpha^{*}$ and $l^{*}$ hence depend on the parameters $\lambda, \sigma^{2}$ and $c$ only through $b=2 \lambda \sigma^{2} c$. It is easily verified that the minimizing $\alpha^{*}$ tends to 0 as $b \rightarrow 0$ and tends to $\frac{1}{2}$ as $b \rightarrow \infty$. In particular it follows that for any given values of two of the parameters $\lambda, \sigma^{2}$ and $c$ and any prespecified $0<\alpha^{*}<\frac{1}{2}$, there exists a value of the third parameter for which the minimizer of $l(x)$ is the prespecified $\alpha^{*}$.

(2) The constrained version. An alternative and natural presentation of the dynamic sampling problem is one in which the average long-run sampling rate is constrained to be at some specified level $\gamma>0$ and the problem is to minimize the average long-run error rate subject to this constraint. Using the preceding arguments, it follows directly that the optimal solution to this constrained problem has the same $U(M, \varepsilon)(M \rightarrow \infty, \varepsilon \rightarrow 0)$ sampling policy but the numerical evaluation of the critical level $\alpha^{*}$ should be adjusted as follows. First rewrite (4.1) as

$$
l(x)=x+c \gamma(x) .
$$

Note that by the analysis of Section $3, l(x)$ is the long-run average cost per unit time when applying the $U(M, \varepsilon)(M \rightarrow \infty, \varepsilon \rightarrow 0)$ policy, but with $\alpha^{*}$ replaced by $x\left(0<x<\frac{1}{2}\right)$. Equation (4.2) splits this cost into its two components, $x$, which is the error level and $\gamma(x)$, which is the average sampling rate [see in particular (3.24) and the arguments there]. 
For the constrained version of the problem we choose $\alpha^{*}$ as the solution to the equation $\gamma(x)=\gamma$. Since (as can be seen from previous arguments, or verified directly), $\gamma(x)$ is strictly monotone over $\left(0, \frac{1}{2}\right)$ with $\gamma(x) \rightarrow \infty$ as $x \rightarrow 0$ and $\gamma(x) \rightarrow 0$ as $x \rightarrow \frac{1}{2}$, it follows that for any prespecified sampling level $\gamma>0$, there exists a unique $0<\alpha^{*}<\frac{1}{2}$ solving $\gamma\left(\alpha^{*}\right)=\gamma$. This $\alpha^{*}$ is the average infimal long-run average error rate for the constrained problem. It is also the $\alpha^{*}$ for the original problem with $\operatorname{cost} c$ for a properly chosen $c$ [see part (1) of this section].

(3) Relative efficiency. It is of course of major interest to compare the dynamic sampling procedure to the procedure in which the sampling rate is fixed. The comparison is more natural when considering the constrained version for the dynamic sampling case.

For the fixed-rate problem, it is shown in Liptser and Shiryayev (1977) that $X(t)$ has a stationary density given by

$$
q(x)=\frac{k}{x^{2}(1-x)^{2}} \exp \left(-2 \lambda \sigma^{2}\left(\frac{x}{1-x}+\frac{1-x}{x}\right)\right),
$$

for $0<x<1$, where $k$ is a normalizing constant. Using symmetry, the longrun average error rate for the fixed rate case, denoted $\alpha$ (f.r.), is hence given by

$$
a(\text { f.r. })=2 \int_{0}^{1 / 2} x \cdot q(x) d x .
$$

It is also shown that as $\lambda \sigma^{2} \rightarrow 0$, the value in (4.4) is (approximately) given by

$$
\alpha(\text { f.r. }) \cong-2 \lambda \sigma^{2} \ln \left(\lambda \sigma^{2}\right) .
$$

For the dynamic sampling procedure in its constrained form, we solve the equation $\gamma(\alpha)=\gamma$, namely

$$
2 \lambda \sigma^{2}(1-2 \alpha)\left[2 \ln \left(\frac{1-\alpha}{\alpha}\right)+\frac{1-2 \alpha}{\alpha(1-\alpha)}\right]=\gamma
$$

and the $\alpha^{*}$ solving (4.6) is the long-run average error rate for the dynamic sampling problem, denoted next by $\alpha$ (d.s.) The value of $\alpha($ d.s. $)$ is seen to depend on $\lambda, \sigma^{2}$ and $\gamma$ only through $\gamma /\left(2 \lambda \sigma^{2}\right)$. As $\lambda \sigma^{2}$ tends to $0, \alpha($ d.s. $)$ also tends to 0 and from (4.6) it is given approximately by

$$
\alpha(\text { d.s. }) \cong \frac{2 \lambda \sigma^{2}}{\gamma} .
$$

Two types of comparison appear natural in this context. The first compares $\alpha$ (f.r.) to $\alpha$ (d.s.) with the same average sampling rate. Substituting $\gamma=1$ in (4.7) yields

$$
\frac{\alpha \text { (d.s. })}{\alpha \text { (f.r. })} \cong \frac{-1}{\ln \left(\lambda \sigma^{2}\right)},
$$

which tends to 0 as $\lambda \sigma^{2} \rightarrow 0$. 
TABLE 1

Numerical results for various values of $\lambda \sigma^{2} 1$

\begin{tabular}{lccccllll}
\hline $\boldsymbol{\lambda} \boldsymbol{\sigma}^{2}$ & $\mathbf{1}$ & $\mathbf{0 . 5}$ & $\mathbf{0 . 1}$ & $\mathbf{0 . 0 5}$ & $\mathbf{0 . 0 1}$ & $\mathbf{0 . 0 0 1}$ & $\mathbf{0 . 0 0 0 1}$ & $\mathbf{0 . 0 0 0 0 1}$ \\
\hline$\alpha$ (f.r.) & 0.403 & 0.367 & 0.246 & 0.186 & 0.0750 & 0.0130 & $0.00184^{2}$ & $0.000230^{2}$ \\
$\alpha$ (d.s.) & 0.378 & 0.330 & 0.174 & 0.105 & 0.0219 & 0.00204 & 0.000201 & 0.0000200 \\
$\gamma$ & 0.616 & 0.598 & 0.506 & 0.444 & 0.294 & 0.165 & $0.110^{2}$ & $0.0871^{2}$ \\
\hline
\end{tabular}

${ }^{1}$ All numbers are rounded up to three significant digits.

${ }^{2}$ Computed using the approximation in equation (4.5).

A second comparison may be made by equating the error rates and solving for $\gamma$. This yields

$$
\gamma \cong \frac{-1}{\ln \left(\lambda \sigma^{2}\right)},
$$

which again tends to 0 as $\lambda \sigma^{2} \rightarrow 0$, indicating that the dynamic sampling procedure can achieve the same error rate with only a fraction of the sampling time.

Numerical results for various values of $\lambda \sigma^{2}$ are summarized in Table 1. Thus for example, when $\lambda \sigma^{2}=0.001$, the error rate for the fixed rate sampling is about 1.3 percent while being only about 0.2 percent with dynamic sampling. To maintain the same 1.3 percent error rate, the dynamic sampling procedure needs less than 17 percent of the sampling amount needed by the fixed rate method.

The comparison also clarifies another aspect of the basic difference between the fixed rate and dynamic sampling procedures. Assume there has been a decision to allow an increase in sampling effort by, say, 30 percent. Evidently one can obtain improved performance by either of the methods. In fixed rate sampling this would come into effect by decreasing the value of $\sigma^{2}$ to $\sigma^{2} / 1.3$ resulting in corresponding values of (4.3), (4.4) and (4.5). The dynamic sampling approach is solving (4.6) with $\gamma$ replaced by $1.3 \gamma$ and moving the critical level to the lower resulting value $\alpha$ (d.s.), which solves the corresponding equation.

5. Additional comments. (1) Applying the $U(M, \varepsilon)$ sampling policy in the limiting $M \rightarrow \infty, \varepsilon \rightarrow 0$ sense causes the $X$-process to appear as if it occasionally shifts "in no time" between the two states $\alpha^{*}$ and $1-\alpha^{*}$ (excluding perhaps an initial transient period). Using the estimation rule $\hat{Y}^{*}$, the error probability is hence constant at $\alpha^{*}$.

(2) Several well-known models are closely related to the one studied in this article. One of these is the problem of search for a target which moves between several locations according to a specified Markov process, and the objective is to search the locations until the target is detected, using the minimal expected total cost. The problem is studied by Weber (1986), while the dynamic search version is studied by Assaf and Sharlin-Bilitzky (1994). A second class of models are quality control problems in general and the change point detec- 
tion problem in particular. It seems that the first to discuss the possibility of controlling the variance is Bather (1976). Dynamic sampling for the change point problem is suggested and analyzed by Assaf (1988).

(3) The sufficiency of the optimality condition (3.5) is fairly straightforward to prove directly but the proof is omitted here since it appears to be well known and has been proven in many analogous cases [see for example Fleming and Rishel (1975), Gihman and Skorohod (1979), as well as Ross (1982) for the discrete case]. Inequality (3.5) also proves optimality of $\hat{Y}^{*}$ since the term $\min \{x, 1-x\}$ is the lowest error rate possible at state $x$. The following comments 4 and 5 address other insights regarding the optimality conditions.

(4) The more familiar form of the optimality conditions for the average cost case is perhaps the equality (as $\Delta t \rightarrow 0$ )

$$
G(x)+l \Delta t=\inf _{u}\left\{c(x, u) \Delta t+E_{u} G(x+\Delta X)\right\},
$$

where $u$ is a general "action," $c(x, u)$ is the cost rate associated with state $x$ and action $u$, and $\Delta X$ is the change of state during the time $\Delta t$ with expectation $E_{u}$ depending on the action $u$. If equality holds (for all $x$ ) for some admissible function $G$, then $l$ is the infimal long-run average cost rate. Inequality in (5.1) ensures that such an $l$ is a lower bound on the infimal rate. Taking (5.1) with inequality as $\Delta t \rightarrow 0$ in our problem results in (3.5).

(5) A constructive method of arriving at an appropriate function $G(x)$ is by taking it to be the difference, in accumulated loss, between starting the problem at state $x$ or at some "nominal" state ( $\alpha^{*}$ is selected for our problem) when using an optimal policy. It may be verified that $G(x)$ defined in (3.13) has this property when the $U(M, \varepsilon)$ sampling policy is applied in the limiting sense and symmetry around $\frac{1}{2}$ is used.

(6) With constant rate sampling, "real time" and "sampling time" are identical (assuming that the constant sampling rate is 1. Otherwise they are proportional). Dynamic sampling allows separating them. The $U(M, \varepsilon)$ policies indicate, in fact, that it is best to try and separate them as much as possible.

(7) Extensions of the model to more than two states and/or nonsymmetric cases result in severe technical difficulties. This is the case for the basic model [as indicated by Yao (1985)]. The dynamic sampling extensions appear to be extremely difficult to handle analytically as well, although there seems to be hope for some extensions in the future.

(8) A basic assumption made in this article is that multiplying the sampling rate by $u$ results in a noise variance of $\sigma^{2} / u$. Taking $u=2$ as an example may be interpreted as having two parallel independent samples of size $\Delta t$, each having variance of $\sigma^{2} \cdot \Delta t$ for estimating the mean. Averaging the two estimators hence results in an estimator with variance $\left(\sigma^{2} / 2\right) \Delta t$. The interpretation is precise when the theoretical sampling ability is unlimited. If the sampling rate is bounded by some number $M_{0}$ then a similar analysis shows that the $U\left(M_{0}, \varepsilon\right)$ policies are optimal when $\varepsilon \rightarrow 0$. The analysis is more delicate in this case since the quantities (3.30), (3.31) and (3.32) for the first part of the cycle need to be written more explicitly in this case. Depending on the partic- 
ular application, one may also need to take a correction for finite population into account when setting up the model.

(9) Some of the small error rates in Table 1 may appear somewhat artificial. These, however, naturally depend on the particular application. In screening for contaminated populations, for example, small error rates are desirable, and the difference between $10^{-3}$ and $10^{-4}$ may be of utmost importance.

(10) The error rate in (4.7) is also obtained by Yao (1985) for the same problem (without dynamic sampling), but when smoothing is used rather than filtering. This is surprising and some further research is called for to check the relation (if any) between the two methods.

\section{REFERENCES}

ASSAF, D. (1988). A dynamic sampling approach for detecting a change in distribution. Ann. Statist. 16 236-253.

Assaf, D. and Sharlin-Bilitzky, A. (1994). Dynamic search for a moving target. J. Appl. Probab. 31 438-457.

BATHER, J. A. (1976). A control chart model and a generalized stopping problem for Brownian motion. Math. Oper. Res. 1 209-224.

Fleming, W. H. and Rishel, R. W. (1975). Deterministic and Stochastic Optimal Control. Springer, New York.

Gihman, I. I. and Skorohod, A. V. (1979). Controlled Stochastic Process. Springer, New York.

Karlin, S. and TAYlor, H. M. (1981). A Second Course in Stochastic Processes. Academic Press, New York.

KHASMinskit, K. Z. and LAZAREVA, B. V. (1992). On some filteration procedure for jump Markov process observed in white Gaussian noise. Ann. Statist. 20 2153-2160.

LIPTSER, R. S. and SHIRYAYEV, A. N. (1977). Statistics of Random Processes. Springer, New York. Ross, S. M. (1982). Introduction to Stochastic Dynamic Programming. Academic Press, New York. Ross, S. M. (1983). Stochastic Processes. Wiley, New York.

WEBER, R. R. (1986). Optimal search for a randomly moving object. J. Appl. Probab. 23 708-717. YAO, Y-C. (1985). Estimation of noisy telegraph processes: nonlinear filtering versus nonlinear smoothing. IEEE Trans. Inform. It-31 444-446.

DEPARTMENT OF STATISTICS

THE HEBREW UNIVERSITY

JERUSALEM 91905

ISRAEL

E-MAIL: msdave@pluto.mscc.huji.ac.il 